\title{
Peppermint Oil to Improve Visualization in Screening Colonoscopy: A Randomized Controlled Clinical Trial
}

\author{
Ishani Shah ${ }^{\mathrm{a}}$, Noemi J. Baffy ${ }^{\mathrm{b}}$, Jennifer L. Horsley-Silva ${ }^{\mathrm{b}}$, \\ Blake T. Langlais ${ }^{c}$, Kevin C. Ruff ${ }^{\text {b, d }}$
}

\begin{abstract}
Background: Screening colonoscopy has been associated with reduced mortality from colorectal cancer by means of early detection and timely treatment. However, visualization during colonoscopy is often impaired since the colon is naturally prone to peristalsis and spasm. There is evidence to suggest benefit of topical peppermint oil in causing smooth muscle relaxation, thereby decreasing peristalsis. The aim of our study was to determine if peppermint oil helps reduce colonic spasticity so as to allow for better visualization during screening colonoscopy.
\end{abstract}

Methods: We performed a randomized controlled, double-blinded, clinical trial where patients undergoing screening colonoscopy were assigned to receive either peppermint oil or placebo. Once cecum was reached, $50 \mathrm{~mL}$ of either solution was directly injected via the working channel of the colonoscope. Colonic peristalsis, spasticity and bowel visibility were documented. Bowel preparation quality, withdrawal time and adenoma detection rate (ADR) were also assessed. Continuous variables were analyzed using $t$-test or Wilcoxon rank-sum test while categorical variables were compared using the two-way Chi-square test.

Results: Forty-eight patients were included, of whom 24 patients received peppermint oil and 24 received placebo. Mean Boston bowel preparation score (BBPS) was excellent for both groups (8 points vs. 7.9 points; $\mathrm{P}=0.98)$. Both mean total colonoscopy time $(17.8 \mathrm{~min}$ vs. $21.9 \mathrm{~min} ; \mathrm{P}=0.07)$ and mean cecal intubation time (7.2 min vs. $10.3 \mathrm{~min} ; \mathrm{P}=0.04$ ) were shorter with peppermint oil as compared to placebo. Complete absence of bowel spasticity was observed among $58.3 \%$ patients in the peppermint oil group as compared to $45.8 \%$ patients in the placebo group ( $\mathrm{P}=0.05)$. More than $75 \%$ of bowel was visualized in $83 \%$ of patients in both groups $(\mathrm{P}=0.56)$. Mean ADR was higher in the peppermint group as compared to the placebo group

Manuscript submitted April 5, 2019, accepted April 17, 2019

a Department of Internal Medicine, Creighton University St. Joseph's Hospital and Medical Center, Phoenix, AZ, USA

bDepartment of Gastroenterology, Mayo Clinic, Scottsdale, AZ, USA

'Department of Biostatistics, Mayo Clinic, Scottsdale, AZ, USA

${ }^{\mathrm{d} C o r r e s p o n d i n g ~ A u t h o r: ~ K e v i n ~ C . ~ R u f f, ~ D e p a r t m e n t ~ o f ~ G a s t r o e n t e r o l o g y, ~}$

Mayo Clinic, 13400 E Shea Blvd, Scottsdale, AZ 85259, USA.

Email: Ruff.Kevin@mayo.edu

doi: https://doi.org/10.14740/gr1180
(45.8\% vs. $37.5 \% ; \mathrm{P}=0.56)$.

Conclusion: Our study suggests that topical peppermint oil reduces bowel wall spasticity, which could lead to better visualization of the bowel during screening colonoscopy. Although use of peppermint oil was associated with better ADRs, these results did not achieve statistical significance. Larger sample size and use of alternative methods of peppermint oil administration allowing for more absorption time may establish stronger results.

Keywords: Peppermint oil; Colonoscopy; Colorectal neoplasms; Peristalsis

\section{Introduction}

Colorectal cancer (CRC) is the third most common type of cancer and also the third leading cause of death in the USA [1]. Colonoscopy is the standard test of choice for CRC screening and has been associated with reduced mortality rates from CRC by detection and removal of tumors and premalignant lesions at an earlier and more treatable stage [2].

Adenoma detection rate (ADR) is a quality measure index that is defined as the proportion of screening colonoscopies performed to detect at least one histologically confirmed adenoma or adenocarcinoma [3]. Many techniques are being developed and evaluated to increase ADRs with the ultimate goal being improved qualitative outcomes associated with screening colonoscopy. One such method involves administration of an antispasmodic agent before the procedure. With the colon being naturally prone to peristalsis and spasmodic contraction, use of an antispasmodic agent is believed to help with colonic relaxation, thereby accounting for better visibility. There is evidence to suggest that topical application of L-menthol, which is an active ingredient of peppermint oil, a plant-based derivative, helps with reduction in colonic spasticity by means of its smooth muscle relaxant properties. This effect has been postulated to enhance bowel wall visualization with subsequent improvement in ADRs [4].

The aim of this study was to determine if topical application of peppermint oil during screening colonoscopy helps with better visualization by means of causing smooth muscle relaxation in the colonic wall and thereby reducing colonic spasticity. We sought to assess measures of colonic spastic- 
Table 1. Classification of Severity of Colonic Peristalsis as Subjectively Graded by Endoscopists in Our Study

\begin{tabular}{lll}
\hline Grade & Severity of peristalsis & Subjective endoscopic description \\
\hline Grade 0 & No peristalsis & No movement observed, with colon dilated \\
Grade 1 & Mild peristalsis & Colon movement not observed, but haustral septae with mild spasm remained \\
Grade 2 & Moderate peristalsis & Colon movement \\
Grade 3 & Severe peristalsis & Severe spasm \\
\hline
\end{tabular}

ity and peristalsis, degree of bowel wall visibility, impact on ADRs of the endoscopists and overall patient satisfaction with the use of peppermint oil for screening colonoscopy.

\section{Materials and Methods}

\section{Patients and study design}

Our study was a randomized controlled, double-blinded, clinical trial. Patients $\geq 50$ years of age undergoing an initial screening colonoscopy were considered eligible for the study. Those who were unable to provide consent had a known allergy to peppermint oil or menthol, had an established diagnosis of inflammatory bowel disease, uncorrectable coagulopathy or renal impairment, as well as those who were pregnant, lactating or incarcerated at the time of colonoscopy were not considered eligible for enrollment. Patients were later excluded from the intervention if the cecum was not reached or the bowel preparation was inadequate. Enrolled participants were randomly assigned to either receive peppermint oil or placebo. Allocation was assigned via using a randomization table, generated by independent staff members just before the examination. Blinding was aided by supplementing all of the participating procedure rooms with peppermint oil saturated gauze. We used the CONSORT guidelines for adequate reporting of our randomized controlled trial [5].

\section{Endoscopic procedure}

On the night before procedure, patients underwent bowel cleansing via consuming a split-dose of GoLytely ${ }^{\circledR}$ (Braintree Laboratories Inc., Braintree, MA, USA) or MoviPrep ${ }^{\circledR}$ (Salix Pharmaceuticals, Raleigh, NC, USA) bowel preparation products. Conscious sedation for the procedure was titrated at the endoscopists' discretion in an outpatient endoscopy center. The colonoscopic examination was performed using adult or

Table 2. Extent of the Total Bowel Wall Visualized, as Subjectively Graded by Endoscopists in Our Study

\begin{tabular}{ll}
\hline Grade & Extent of total bowel wall visualized (\%) \\
\hline Grade 0 & $\geq 75 \%$ \\
Grade 1 & $50-75 \%$ \\
Grade 2 & $25-49 \%$ \\
Grade 3 & $\leq 25 \%$ \\
\hline
\end{tabular}

pediatric colonoscopes (Olympus ${ }^{\circledR}$ PCF-H190L or Olympus ${ }^{\circledR}$ CF-HQ190L).

The peppermint oil solution and placebo were prepared on site by the pharmacy department. An $8 \mathrm{~mL}$ volume of peppermint oil (Humco ${ }^{\circledR}$, Texarkana, TX, USA) and $0.2 \mathrm{~mL}$ of Tween 80 , a non-ionic surfactant $\left(\right.$ Medisca $^{\circledR}$, Las Vegas, NV, USA) were gently mixed and dissolved in $42 \mathrm{~mL}$ of distilled water. The placebo solution was constituted from $0.4 \mathrm{~mL}$ of Maalox ${ }^{\circledR}$ (mixture of aluminum hydroxide, magnesium hydroxide and simethicone) mixed with $49 \mathrm{~mL}$ of distilled water. Both solutions were compounded on the day of the procedure. The placebo was similar to the peppermint oil in terms of consistency, color and appearance.

All colonoscopies were performed as per the standard practice of first delivering the colonoscope to the cecum without looking for lesions. Then, $50 \mathrm{~mL}$ of the prepared solution in a prefilled syringe, either peppermint oil or placebo, was gently delivered into the cecum and directly sprayed on the bowel wall through the working channel of the colonoscope. The working channel was then flushed with air to ensure that no residual fluid remained in the colonoscope. During the procedure, heart rate and pulse oximetry were monitored continuously in all patients. The endoscopists subjectively graded the overall severity of colonic peristalsis, extent of total bowel wall visualized, and degree of colonic wall spasticity using the classifications described in Tables 1, 2 and 3, respectively.

\section{Endpoints}

Baseline information regarding demographics, anthropometrics and medication utilization were recorded. Procedural details such as cecal intubation time, colonoscopic withdrawal time and total procedure time were noted. Additional data on quality measures such as bowel preparation quality using the Boston bowel preparation score (BBPS) as well as visualization and spasticity parameters (described in Tables 1,2 and

Table 3. Degree of Colonic Wall Spasticity, as Subjectively Graded by Endoscopists in Our Study

\begin{tabular}{ll}
\hline Grade & Degree of colonic wall spasticity \\
\hline Grade 0 & $\geq 75 \%$ \\
Grade 1 & $50-75 \%$ \\
Grade 2 & $25-49 \%$ \\
Grade 3 & $\leq 25 \%$ \\
Grade 4 & No spasticity observed \\
\hline
\end{tabular}


Table 4. Comparison of Baseline Patient Characteristics Between Peppermint Oil and Placebo Groups

\begin{tabular}{llll}
\hline Patient characteristics & Peppermint oil group $(\mathbf{N}=\mathbf{2 4})$ & Placebo group $(\mathbf{N}=\mathbf{2 4})$ & P-value \\
\hline Mean age (years) & $53.8 \pm 3.9$ & $54.8 \pm 4.9$ & 0.41 \\
BMI $\left(\mathrm{kg} / \mathrm{m}^{2}\right)$ & $29.6 \pm 5.3$ & $26.3 \pm 5.8$ & 0.02 \\
Gender, n (\%) & & & 0.07 \\
$\quad$ Male & $13(54.2 \%)$ & $5(20.8 \%)$ & $19(79.2 \%)$ \\
$\quad 11(45.8 \%)$ & $2(8.3 \%)$ & 1 \\
Female & $2(8.3 \%)$ & $2(8.3 \%)$ & 0.15 \\
\hline Chrobetes mellitus, $\mathrm{n}(\%)$ & $0(0 \%)$ & & \\
\hline
\end{tabular}

3) were collected. The location, size and macroscopic as well as histologic types of all detected lesions were documented. ADRs were computed. Soon after completion of the colonoscopy, all enrolled patients were asked to report their perception of pain during the procedure using a visual analogue scale. They were also asked to rate their willingness to repeat colonoscopy in the future on a five-point Likert scale.

\section{Data collection and statistical analysis}

Study data were collected using REDCap ${ }^{\circledR}$ (Research Electronic Data Capture), an electronic data capture tool, hosted securely at Mayo Clinic [6]. Continuous variables were expressed as means and standard deviations. These were compared between the peppermint oil and placebo groups using $t$-test or Wilcoxon rank-sum test as deemed appropriate. Categorical variables were expressed as frequencies and were compared between the two groups using the two-way Chi-square test. Statistical significance was assigned at 0.05 .

\section{Ethical considerations}

This study was approved by the Institutional Review Board (IRB) at Mayo Clinic, Arizona. It was conducted in compliance with ethical standards of Mayo Clinic on human subjects as well as with the 1964 Helsinki Declaration [7]. The trial was registered with Clinicaltrials.gov under the identifier NCT03286764.

\section{Results}

A total of 51 patients initially met eligibility criteria for our study. Of these, two patients withdrew from the study after signing consent while one patient had an inadequate bowel preparation and so the colonoscopy was rescheduled. These three patients were, therefore, excluded from the study.

Forty-eight patients successfully completed the study with 24 patients randomly enrolled in each arm of the study, that is, 24 patients received peppermint oil and 24 patients received placebo by randomization. These 48 colonoscopies were performed by 10 skilled endoscopists over a period of 4 weeks in an open access endoscopy unit at the Mayo Clinic in Scotts- dale, Arizona.

Demographic characteristics of patients in both groups are outlined in Table 4. Average age of the patients enrolled in the study was 54.3 years with no significant difference between the two groups. The peppermint group had fewer females (45.8\% vs. $79.2 \% ; \mathrm{P}=0.07)$ as compared to the placebo group. Presence of predisposing factors for poor bowel preparation prior to colonoscopy, such as diabetes mellitus $(8.3 \%$ in both groups; $\mathrm{P}=1)$ and use of chronic opioids ( $0 \%$ vs. $8.3 \% ; \mathrm{P}=$ $0.15)$ was similar between the two groups.

MoviPrep ${ }^{\circledR}$ was the favored purgative $(66.7 \%)$ in both groups $(\mathrm{P}=1)$. The BBPS was documented ( 8 vs. $7.9 ; \mathrm{P}=0.98$ ) and was consistent with excellent bowel preparation quality in both groups. Total colonoscopy time with peppermint oil was $17.8 \mathrm{~min}$ as compared to $21.9 \mathrm{~min}$ in the placebo group $(\mathrm{P}=$ $0.07)$. Similarly, withdrawal time in the peppermint oil group was $10.6 \mathrm{~min}$ as compared to $11.6 \mathrm{~min}$ in the placebo group $(P=0.43)$. Average time to reach the cecum was lesser in the peppermint oil group compared to the placebo group (7.2 min vs. $10.3 \mathrm{~min} ; \mathrm{P}=0.04)$. Visualization parameters were determined by the endoscopists on withdrawal of the colonoscope and colonic spasticity was considerably less prominent when peppermint oil was used as compared to placebo $(\mathrm{P}=0.05)$. Other procedural details and visualization parameters as determined by the endoscopists are outlined in Table 5 .

Peppermint oil was generally well tolerated, with no adverse events or symptoms reported during or after the procedure. Patient's perception of pain was generally low with no difference between the treatment arms. A larger proportion of patients $(62.5 \%$ vs. $43.5 \% ; \mathrm{P}=0.30)$ in the treatment arm with peppermint oil rated their willingness to repeat the colonoscopy as "very likely". However, there were overall high rates of patient satisfaction seen in both groups (Table 5).

\section{Discussion}

Worldwide, approximately 1.2 million people are diagnosed annually with $\mathrm{CRC}$, and the World Health Organization estimates an increase of $77 \%$ in the number of newly diagnosed cases of CRC by 2030 [8]. Consequently, a good quality screening colonoscopy is our best tool to identify and remove adenomatous polyps and other premalignant lesions in a timely manner, in an effort to reduce CRC incidence and mortality.

With ADRs and 6-min colonoscopy withdrawal time hav- 
Table 5. Comparison of Procedural Details and Visual Parameters Between Peppermint Oil and Placebo Groups

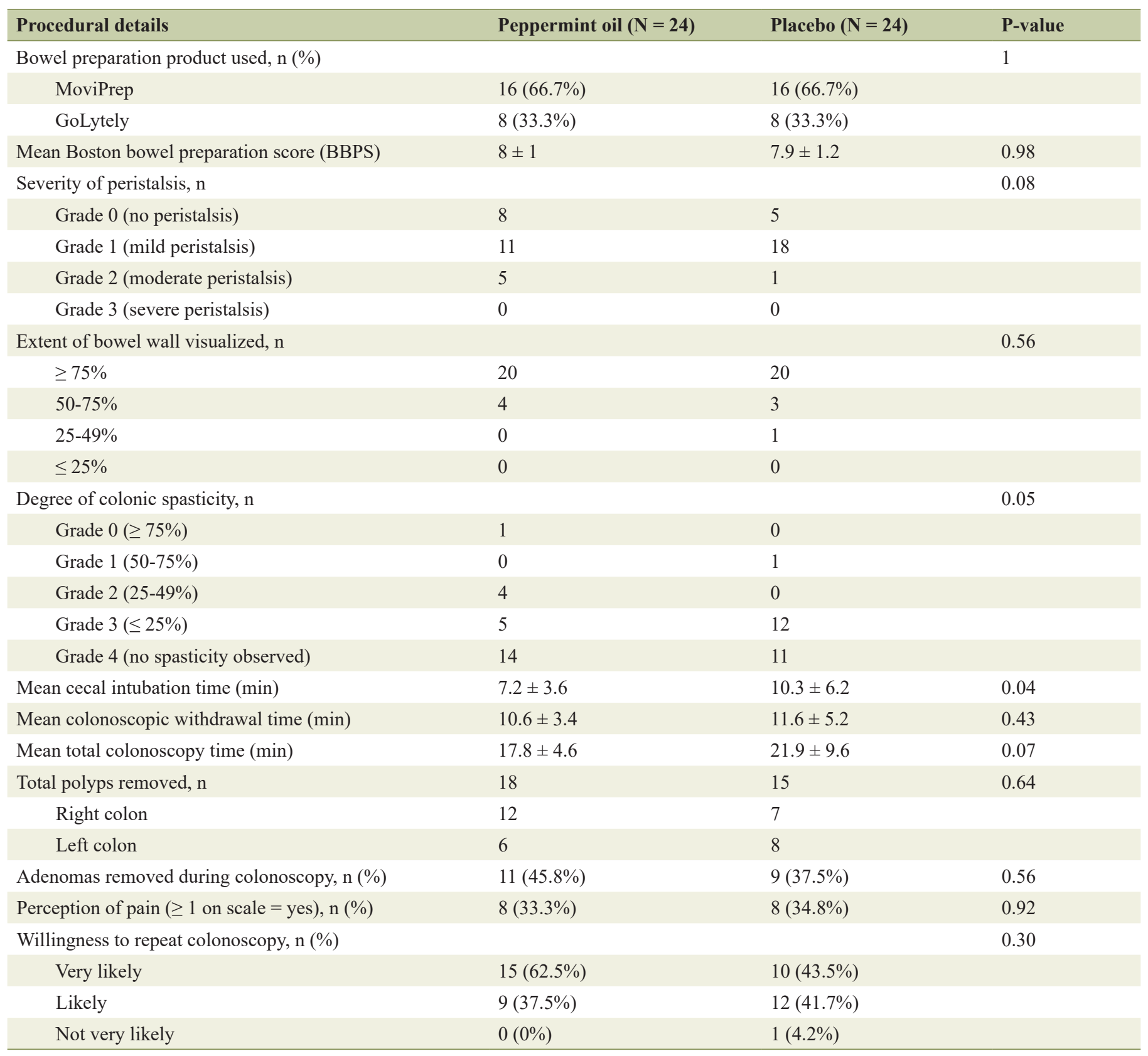

ing emerged as important quality measures to ensure thorough colonic examination during the procedure, many techniques are being developed to enhance colonic visibility during the procedure. These include introduction of high-definition white light, visual image enhancement technologies, cap-assisted colonoscopy and use of water immersion or exchange techniques [9]. A simpler method described to increase ADR is the administration of an antispasmodic agent before the procedure, effectively decreasing peristalsis and providing better visualization of the colonic mucosa. Antispasmodic agents such as hyoscine butyl bromide and glucagon have been used in intravenous forms prior to the procedure to inhibit peristalsis during colonoscopy [10]. However, systemic administration of these agents has been associated with adverse reactions such as cardiovascular complications and delayed hypoglycemia after administration of hyoscine butyl bromide and glucagon respectively $[11,12]$. Various other minor side effects have also been reported such as nausea, vomiting and ocular, urinary, and allergic symptoms [13-16]. In the United Kingdom, however, a recent multicenter study demonstrated that use of hyoscine butyl bromide resulted in a global increase in ADR (16-18.1\%) with a larger difference noted in poorer performing colonoscopists [17].

Peppermint oil is an extract derived from the Mentha 
piperita plant, with its main constituent being L-menthol, which is present in a concentration of $30-55 \%$ [18]. Early investigation on peppermint oil in a guinea pig model showed that it exerts its effect on potential-dependent calcium channels by decreasing the peak current amplitudes while also increasing the rate of current decay [19]. An in vitro study on human colon specimens confirmed that menthol exerts its inhibitory effect on smooth muscle contraction by acting as an antagonist at the L-type $\mathrm{Ca}^{2+}$ channels [20]. In the gastrointestinal tract, this blockade of calcium channels leads to relaxation of the smooth muscles and eventual decrease in peristaltic activity [21]. Peppermint oil is generally safe and well-tolerated when taken orally. Some transient side effects have been reported such as abnormal anal and perianal sensation, dry mouth, belching, rash, dizziness, headache and appetite changes [22].

The administration of L-menthol topically in the colon is a simple technique which may improve visibility and thereby, enhance ADRs significantly. A Japanese study group recently showed a $17.6 \%$ increase in ADRs by using topical L-menthol upon cecal intubation in a single-blinded placebo controlled trial [4]. There is also evidence that spraying L-menthol directly on the mucosa is helpful in relaxing the duodenum during endoscopic retrograde cholangiopancreatography procedures and in suppressing gastric peristalsis during upper gastrointestinal endoscopy $[23,24]$. Premedication with enteric coated peppermint oil has been shown to decrease the time required for cecal intubation as well as the total procedure time in addition to reducing colonic spasm, increasing endoscopist satisfaction and decreasing perception of pain in patients during colonoscopy [25]. Enteric coated peppermint oil has also been shown to significantly improve symptoms in irritable bowel syndrome. Individuals taking peppermint oil experienced less severe abdominal pain and distension and had reduced stool frequency, in addition to having less borborygmi and flatulence [22]. Routine use of antispasmodics has, however, not become widespread despite their proven efficacy and relatively safe side effect profile.

Our study shows that use of topical peppermint oil in patients undergoing screening colonoscopy was associated with lesser degrees of colonic peristalsis and hence, spasticity, as compared to placebo. It was also associated with a shorter colonoscopic withdrawal time. The shorter cecal intubation time, however, cannot be attributed to use of peppermint oil, since the solution was flushed into the bowel only after the cecum was reached. However, reduced peristalsis and spasticity in addition to faster colonoscopic withdrawal in patients receiving peppermint oil might have attributed to enhanced visibility, ultimately leading to faster completion of the colonoscopy, as indicated by shorter total procedure time. ADRs were marginally higher among patients receiving peppermint oil; however, these results were not statistically significant.

There are several limitations to our study. This was a pilot study with 48 participants, thus not powered to detect significant differences in adenoma detection rates; rather it was a study to assess feasibility and ease of use. Excellent ADRs have previously been established among the endoscopists at Mayo Clinic, Arizona (44\% overall; $50 \%$ for males and 38\% for females in 2015). This, in addition to excellent bowel preparation achieved among the patients, may account for the minor improvement in ADRs seen with use of peppermint oil. Additionally, due to the outpatient setting of the endoscopy center, 10 different physicians performed the 48 procedures. Assessment of colon peristaltic activity and visualization is a subjective finding and was assessed by the endoscopists themselves, adding variability to the results. However, presence of a large number of endoscopists can also be perceived as strength of the study since every endoscopist, although experienced, is different in terms of skill sets. Some early studies have demonstrated that peppermint oil, when directly administered in the colon had a quick onset of activity, on an average $21.6 \pm 15$ $\mathrm{s}$, and lasted around $20 \mathrm{~min}$ [26]. Based on these results, we used a single administration of $50 \mathrm{~mL}$ peppermint oil sprayed directly on to the cecal wall of our study participants. However, a recent trial comparing topical administration of lidocaine and peppermint oil has shown that both antispasmodics have a relatively short duration of colonic relaxation $(212.5 \mathrm{~s}$ for L-menthol vs. $227 \mathrm{~s}$ for lidocaine) and that rebound spasms occurred more often after use of peppermint oil [27]. This would suggest that topical utilization of peppermint oil on an as-needed basis may be better suited owing to its pharmacologic characteristics.

Larger, double-blinded, randomized controlled trials are needed with different methods of peppermint oil administration (targeted on-demand vs. fixed anatomical sites) to assess its true benefit. Additional evaluation with inexperienced endoscopists such as trainees or those with lower ADRs may also be valuable. An encapsulated triple microsphere formulation of peppermint oil with delayed release of contents in the small bowel is commercially available and this may prove to be a useful adjunct when preparing the bowel for colonoscopy.

In conclusion, our study, albeit limited by its small sample size, suggests a possible role of peppermint oil in colonoscopy to decrease colonic peristalsis and spasticity, in an effort to improve visualization. Our double-blinded study design can be perceived as the biggest strength of the study, as compared to previous studies where adenoma detection may have been positively biased by the unblinded endoscopists [4]. Alternatively, targeted methods of peppermint oil administration may prove to be more useful. More participants are needed to provide substantial evidence for an impact on ADR.

\section{Acknowledgments}

None.

\section{Financial Disclosure}

This research project was entirely funded by Mayo Clinic's internal grant.

\section{Conflict of Interest}

The authors have no financial interests or affiliations with any 
institutions, organizations, or companies relevant to the manuscript.

\section{Informed Consent}

Informed consent was obtained from all patients participating in this study.

\section{Author Contributions}

Ishani Shah: writing, editing and reviewing the manuscript. Noemi J. Baffy: writing and editing the manuscript; performing conceptualization and administration of the study. Jennifer L. Horsley-Silva: performing conceptualization and administration of the study. Blake T. Langlais: performing statistical analysis of the data. Kevin C. Ruff: supervision, conceptualization and administration of the study.

\section{References}

1. Siegel RL, Miller KD, Fedewa SA, Ahnen DJ, Meester RGS, Barzi A, Jemal A. Colorectal cancer statistics, 2017. CA Cancer J Clin. 2017;67(3):177-193.

2. Edwards BK, Ward E, Kohler BA, Eheman C, Zauber AG, Anderson RN, Jemal A, et al. Annual report to the nation on the status of cancer, 1975-2006, featuring colorectal cancer trends and impact of interventions (risk factors, screening, and treatment) to reduce future rates. Cancer. 2010;116(3):544-573.

3. Anderson JC, Butterly LF. Colonoscopy: quality indicators. Clin Transl Gastroenterol. 2015;6:e77.

4. Inoue $\mathrm{K}$, Dohi $\mathrm{O}$, Gen $\mathrm{Y}$, Jo M, Mazaki T, Tokita K, Yoshida N, et al. L-menthol improves adenoma detection rate during colonoscopy: a randomized trial. Endoscopy. 2014;46(3):196-202.

5. Schulz KF, Altman DG, Moher D, CONSORT Group. CONSORT 2010 Statement: updated guidelines for reporting parallel group randomised trials. BMC Med. 2010;8:18.

6. Harris PA, Taylor R, Thielke R, Payne J, Gonzalez N, Conde JG. Research electronic data capture (REDCap)a metadata-driven methodology and workflow process for providing translational research informatics support. J Biomed Inform. 2009;42(2):377-381.

7. Carlson RV, Boyd KM, Webb DJ. The revision of the Declaration of Helsinki: past, present and future. Br J Clin Pharmacol. 2004;57(6):695-713.

8. Binefa G, Rodriguez-Moranta F, Teule A, Medina-Hayas M. Colorectal cancer: from prevention to personalized medicine. World J Gastroenterol. 2014;20(22):67866808.

9. Dik VK, Moons LM, Siersema PD. Endoscopic innovations to increase the adenoma detection rate during colonoscopy. World J Gastroenterol. 2014;20(9):2200-2211.

10. Ashraf I, Ashraf S, Siddique S, Nguyen DL, Choudhary
A, Bechtold ML. Hyoscine for polyp detection during colonoscopy: A meta-analysis and systematic review. World J Gastrointest Endosc. 2014;6(11):549-554.

11. Sanagapalli S, Agnihotri K, Leong R, Corte CJ. Antispasmodic drugs in colonoscopy: a review of their pharmacology, safety and efficacy in improving polyp detection and related outcomes. Therap Adv Gastroenterol. 2017;10(1):101-113.

12. Katoh K, Nomura M, Iga A, Hiasa A, Uehara K, Harada K, Nakaya Y, et al. Comparison of gastric peristalsis inhibition by scopolamine butylbromide and glucagon: evaluation by electrogastrography and analysis of heart rate variability. J Gastroenterol. 2003;38(7):629-635.

13. Hashimoto T, Adachi K, Ishimura N, Hirakawa K, Katsube T, Kurotani A, Hattori S, et al. Safety and efficacy of glucagon as a premedication for upper gastrointestinal endoscopy - a comparative study with butyl scopolamine bromide. Aliment Pharmacol Ther. 2002;16(1):111-118.

14. Gonzalez-Mendiola R, Sanchez Fernandez C, Prieto Montano P, Cuevas M, Cena Delgado M, Sanchez Cano M. Acute urticaria induced by hyoscine butylbromide. Allergy. 2004;59(7):787-788.

15. Ozaslan E, Karakelle N, Ozaslan NG. Hyoscine-N-butylbromide induced ventricular tachycardia during ERCP. J Anaesthesiol Clin Pharmacol. 2014;30(1):118-119.

16. Herskovitz PI, Sendovski U. Severe allergic reaction to intravenous injection of glucagon. Radiology. 1997;202(3):879.

17. Rajasekhar PT, Rees CJ, Bramble MG, Wilson DW, Rutter MD, Saunders BP, Hungin AP, et al. A multicenter pragmatic study of an evidence-based intervention to improve adenoma detection: the Quality Improvement in Colonoscopy (QIC) study. Endoscopy. 2015;47(3):217-224.

18. Grigoleit HG, Grigoleit P. Gastrointestinal clinical pharmacology of peppermint oil. Phytomedicine. 2005;12(8):607-611.

19. Hills JM, Aaronson PI. The mechanism of action of peppermint oil on gastrointestinal smooth muscle. An analysis using patch clamp electrophysiology and isolated tissue pharmacology in rabbit and guinea pig. Gastroenterology. 1991;101(1):55-65.

20. Amato A, Liotta R, Mule F. Effects of menthol on circular smooth muscle of human colon: analysis of the mechanism of action. Eur J Pharmacol. 2014;740:295-301.

21. Micklefield GH, Greving I, May B. Effects of peppermint oil and caraway oil on gastroduodenal motility. Phytother Res. 2000;14(1):20-23.

22. Khanna R, MacDonald JK, Levesque BG. Peppermint oil for the treatment of irritable bowel syndrome: a systematic review and meta-analysis. J Clin Gastroenterol. 2014;48(6):505-512.

23. Yamamoto N, Nakai Y, Sasahira N, Hirano K, Tsujino $\mathrm{T}$, Isayama H, Komatsu Y, et al. Efficacy of peppermint oil as an antispasmodic during endoscopic retrograde cholangiopancreatography. J Gastroenterol Hepatol. 2006;21(9):1394-1398.

24. Hiki N, Kaminishi M, Tanabe S, Fujisaki J, Yoshino J, Iguchi M, Kobayashi H, et al. An open-label, single-arm study assessing the efficacy and safety of L: -menthol 
sprayed onto the gastric mucosa during upper gastrointestinal endoscopy. J Gastroenterol. 2011;46(7):873-882.

25. Shavakhi A, Ardestani SK, Taki M, Goli M, Keshteli AH. Premedication with peppermint oil capsules in colonoscopy: a double blind placebo-controlled randomized trial study. Acta Gastroenterol Belg. 2012;75(3):349-353.

26. Asao T, Mochiki E, Suzuki H, Nakamura J, Hirayama I, Morinaga N, Shoji H, et al. An easy method for the in- traluminal administration of peppermint oil before colonoscopy and its effectiveness in reducing colonic spasm. Gastrointest Endosc. 2001;53(2):172-177.

27. Nemoto D, Utano K, Isohata N, Endo S, Kumamoto K, Koshimizu TA, Lefor A, et al. Topical lidocaine inhibits spasm during colonoscopy: a double-blind, randomized controlled trial (with video). Endosc Int Open. 2017;5(6):E402-E407. 